\title{
A novel branch current flow-based construction of microgrids
}

\author{
Kavitha Sivakumar ${ }^{1}$, Jayashree $\mathbf{R}^{2}$, Karthikeyan Danasagaran ${ }^{3}$ \\ ${ }^{1,2}$ Department of Electrical and Electronics Engineering, B. S. Abdur Rahman Crescent Institute of Science and \\ Technology, Chennai, India \\ ${ }^{3}$ Department of Mechanical Engineering, Pune, India
}

\begin{tabular}{l}
\hline Article Info \\
\hline Article history: \\
Received May 20, 2020 \\
Revised Jul 22, 2020 \\
Accepted Aug 6, 2020 \\
\hline
\end{tabular}

Keywords:

Distributed generation

Microgrid design

Reactive power planning

Reliability assessment

Renewable energy resources

\begin{abstract}
An efficient procedure for defining the boundaries of microgrids in smart distribution systems during distributed generation expansion planning becomes an important consideration in constructing smart grids. A novel approach named "Modified Reverse Current Flow Method" is proposed in this paper to split a large radial distribution system into a required number of self-adequate microgrids. This cluster of microgrids will be capable of utilizing maximum power output of the distributed renewable energy generators and will act as highly reliable zones, during both islanded and grid-connected modes. This method is based on the trend reversal of the flow of current in the various parts of the circuit. This paper uses the practical machine operating curves of the distributed generators to calculate their reactive power output. For the considered distribution system, the proposed method is applied to find the optimal point of operation and the boundaries of microgrids. To bring out the superiority of this novel method, the improvement in reliability indices and economic savings of this method are compared with the results obtained using a similar method available in the literature. This method has several notable merits, namely, increased accuracy in the calculation of annual energy losses and the voltage profile.
\end{abstract}

This is an open access article under the CC BY-SA license.

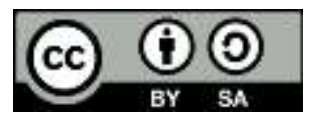

\section{Corresponding Author:}

Kavitha Sivakumar

Department of Electrical and Electronics Engineering

B. S. Abdur Rahman Crescent Institute of Science and Technology

Vandalur, Chennai, India 600048

Email:kavi0705@gmail.com

\section{INTRODUCTION}

In the recent past, it can be seen that a lot of research work has been carried out to redefine the structure of distribution systems, to make them smart. When boundaries of microgrids are defined properly in every distribution zone, decentralized, effectively controllable, and highly reliable smarter distribution zones may be obtained. The problem of defining boundaries of microgrids, when a large distribution system is given, has been solved in the literature using various strategies [1-3]. The authors in [1-3] embedded the concept of distributed generation to a greater extent for a greater self-adequacy, reduced energy losses, and reduced investment cost of the utility. The microgrids also encouraged the installation of renewable energy (RE) distributed generators (DGs) in the customer premises to show reduced emission levels, and improved voltage profile. The current research will inherit these positive attributes of RE DGs, and extend the viewpoint towards increased practicality, by considering the practical P-Q curves of the DGs found in $[4,5]$, to build a cluster of microgrids. When such curves are considered for the formation of microgrids, a more reasonable and highly accurate solution will result. A detailed study of the literature makes it clear that such P-Q curves were never used to calculate the reactive power output of DGs $\left(Q_{D G}\right)$ to build a cluster of microgrids $[1-3,6,7]$. 
Moreover, when the system is split into many numbers of microgrids without any consideration for the minimum number of nodes per microgrid (MNPM) as in [2], it results in the installation of a large number of switches and increases the budget and complexity of the operation. This problem will also be addressed in this paper. When it comes to $100 \%$ utilization of the RE DGs, a new strategy was proposed in [3], considering the uncertainties involved with RE DGs and the loads. The authors in [3] fixed the boundaries of the microgrids based on the real part of the flow of current in the branches $\left(I_{b r}\right)$. However, it is found that that the imaginary part of $I_{b r}$ representing the supply of $Q_{D G}$ is equally important to maintain a good voltage profile and decreased annual energy loss (AEL). The current research thus takes into account this shortcoming of [3] and will arrive at a better outcome when compared to [3].

When the percentage of penetration (PP) of the DGs is exceptionally high or very low as in [3], the operation of the cluster of microgrids will be highly reliant on the substation. In such a case, the isolated operation of a microgrid will result in reduced reliability of the system. This issue found in [3] will also be addressed in the current research. References $[8,9]$ list out the various standard measurement indices to find out the reliability of the microgrids or the distribution system as a whole. The most widely recognized customer-based indices for distribution systems are system average interruption frequency index (SAIFI), and system average interruption duration index (SAIDI). As the current-day distribution systems include numerous DGs installed, some papers in the literature has calculated loss of load probability (LOLP) which was previously used only for the generation segment of the system and not for the distribution module to ascertain reliability [10]. This paper will calculate all the reliability indices and compare them with the case found in the literature to bring out the proficiency of the current work.

The main contributions of this paper are as follows:

a) The development of the novel method based on the flow of current in the branches to construct optimal microgrids that are profoundly self-adequate, exceptionally reliable and are capable of utilizing the maximum generation from the DGs. Practical P-Q curves of the DGs will be used to find reactive power.

b) Finding the best level of infiltration of the distributed generation for the considered system that corresponds to minimum annual energy loss and a maximum number of microgrids.

c) Evaluating the self-adequacy of the cluster of microgrids in terms of $A E L$ and improvement in voltage profile, for varying percentages of penetration of the DGs. Measuring the reliability of the cluster of microgrids in terms of load-based indices, customer-based indices, and LOLP.

\section{THE PROPOSED METHOD}

The conceptual representation of the proposed novel method named as "Modified Reverse Current Flow Method (MRCFM)" is shown in Figure 1. For a particular year, considering the various uncertainties associated with fluctuating load and generation as in [11, 12], there will be numerous mixes of loadgeneration occurring at any given point of time. When an up-to-down flow of $I_{b r}$ occurs in a continuous set of branches with an unexpected change in the progression of current (down-to-up) in an adjacent branch after a node, the node is marked. The branches associated with this marked node become the candidate branches to form the microgrids. This trend reversal of current must hold good for a majority of the cases for the given year. The same logic holds true for a down-to-up flow of $I_{b r}$ in a continuous set of branches.

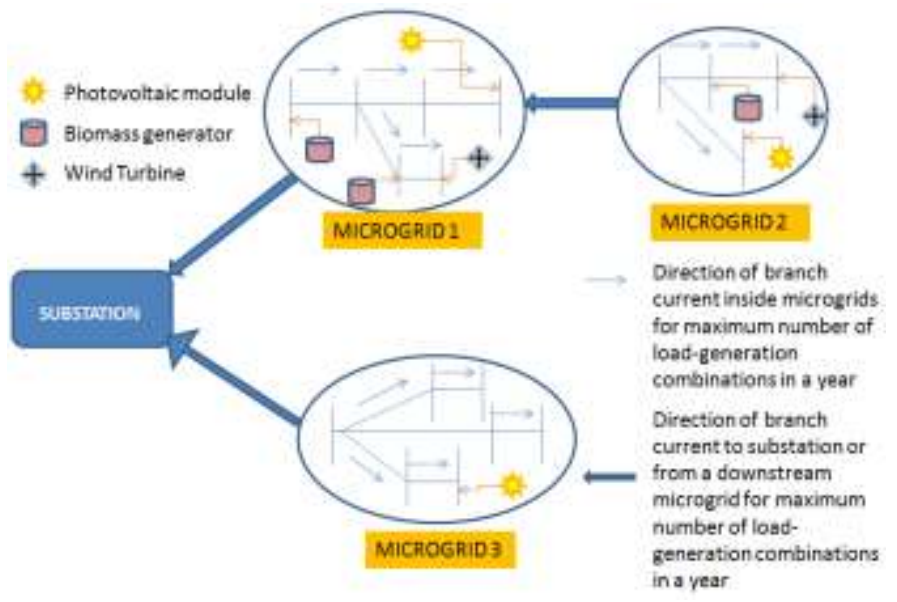

Figure 1. Design concept 


\section{RESEARCH METHOD}

\subsection{Modeling of load and distributed generators}

The hourly load shape of the IEEE-RTS is referred here, and the load is represented using ten discrete levels as in [13]. Utilizing the historical data, the wind speed is modeled using the rayleigh probability density function (PDF) and the solar irradiance is modeled using Beta PDF as in [14-16]. The $P_{D G}$ of the doubly-fed induction generator (DFIG)-based wind systems and the voltage source inverter (VSI)based PV systems are determined as in [3]. For a range between leading power factor (pf) of 0.95 and lagging pf of 0.95 , the operational characteristics of a sample DFIG wind park found in Reference [5] is referred, to find $Q_{D G}$. For PV units and for synchronous generator (SG)-based biomass units, $Q_{D G}$ is calculated as in [4]. The DGs either supply $Q_{D G}$ or absorb the same, based on capacitive or inductive pf, respectively.

\subsection{Combined load-generation modeling}

The operating point of the wind turbines is assumed to lie on the operating curves corresponding to the slip values, -0.25 or -0.05 or 0.25 [5]. On discretizing the output of the wind turbines and PV units, the values of $P_{D G}$ are assumed to be $100 \%$ or $50 \%$ or $0 \%$ of the nominal DG capacity. Thus, when three distinctive operational curves are relating to various estimations of the slip, three diverse percentages of $P_{D G}$ and two different pf (lead or lag); $3 \times 3 \times 2=18$ discrete output states exist for every wind turbine in the system. On merging all the discrete states that do not give output, it can be ended up with 14 distinct stages of output for the wind turbines. Similarly, the count of discrete output states of the PV units equals $3 \times 2=6$, considering the three unique percentages of $P_{D G}$ at lead and lag pf. The probability of occurrence (POC) of each state of the wind turbines is assumed to be 0.055 and that of the solar PV systems is assumed to be 0.167 . Reference [3] gives the corresponding equations. For SG-based biomass generators, the $P_{D G}$ differs from $30 \%$ to $90 \%$ of the nominal capacity of the generator, and Table 1 gives a rundown of the various discrete output levels.

$\underline{\text { Table 1. SG-based biomass generator levels }}$

\begin{tabular}{ccc}
\hline $\begin{array}{c}\text { Generation } \\
\text { Levels }\end{array}$ & $\begin{array}{c}\text { Percentage of } \\
\text { output and pf }\end{array}$ & $\begin{array}{c}\text { Probability of } \\
\text { occurrence }\end{array}$ \\
\hline 1 & $30 \%$ at leading pf & 0.1 \\
2 & $40 \%$ at leading pf & 0.15 \\
3 & $80 \%$ at leading pf & 0.15 \\
4 & $90 \%$ at leading pf & 0.1 \\
5 & $30 \%$ at lagging pf & 0.25 \\
6 & $90 \%$ at lagging pf & 0.25 \\
\hline
\end{tabular}

The POC for any combination of the loads and DGs can be found by using the convolution procedure as given in [16]. The complete annual load-generation model can be found in [3]. The total number of discrete states in the model will come to 5040, considering 14 distinct states of the DFIG-based wind systems, 6 unique states of PV modules, 6 different states of the biomass units, and 10 levels of existence of the loads, in the considered system. It is assumed that the levels of generation are completely independent of the levels of load and that the output of the three different types of generators is independent of each other. Finally, the AEL will be calculated using the formula given in [1].

\subsection{Reliability calculation}

Reference [8] gives the formula and example calculations for the customer-based indices (SAIDI, SAIFI, momentary average interruption frequency index-MAIFI), and the load-based indices (average system interruption frequency index-asifi and average system interruption duration index-ASIDI). When the components undergo a sustained failure in the distribution system, LOLP can be found using (1).

$$
L O L P=\sum_{n=1}^{N O C} \sum_{m=1}^{N O P}\left(S F_{m n} \times R T_{m n} \times L O L E_{m n}\right)
$$

where $N O C$ is the number of components prone to failure in the given system, NOP is the count of the discrete percentages of the $P_{D G}$ in the system, $S F_{m n}$ is the count of sustained failures per hour, $R T_{m n}$ is the repair time in hours for one failure and $L O L E_{m n}$ is the ratio of loss of load in $\mathrm{kVA}$ to total $\mathrm{kVA}$ requirement of the system.

A sustained failure or maintenance scheduling results in loss of load and requires a considerable repair time. The total repair time per year (RTPY) can be calculated as in (2) to decide the required manpower to maintain the distribution system. 


$$
R T P Y=\sum_{n=1}^{N O C} \sum_{m=1}^{N O P}\left(S F P Y_{m n} \times R T_{m n} \times M O P Y_{m n} \times M O T_{m n}\right)
$$

where $S F P Y_{m n}$ is the count of sustained failures per year, $M O P Y_{m n}$ is the count of maintenance outages per year and $M O T_{m n}$ is the time taken for maintenance per year.

\subsection{Operational sequence to construct the microgrids and to evaluate reliability}

The sequence of steps of the operation for a given $P P$ of the DGs is recorded beneath:

Step 1: First get the load data, generator data and uncertainties information using the procedure given above. Generate the load-generation combination file. Then get the bus data, branch data, failure rates of all the components along with the respective repair time, maintenance time and, the number of customers or $\mathrm{kVA}$ affected during each fault.

Step 2: Secondly, solve the power flow problem using the forward-backward sweep (FBS) load flow algorithm, just as in $[3,17,18]$. The bus-injection to branch-current (BIBC) and branch-current to busvoltage (BCBV) matrices are framed as in [19]. The flowchart for determination of the boundaries is delineated in Figure 2.

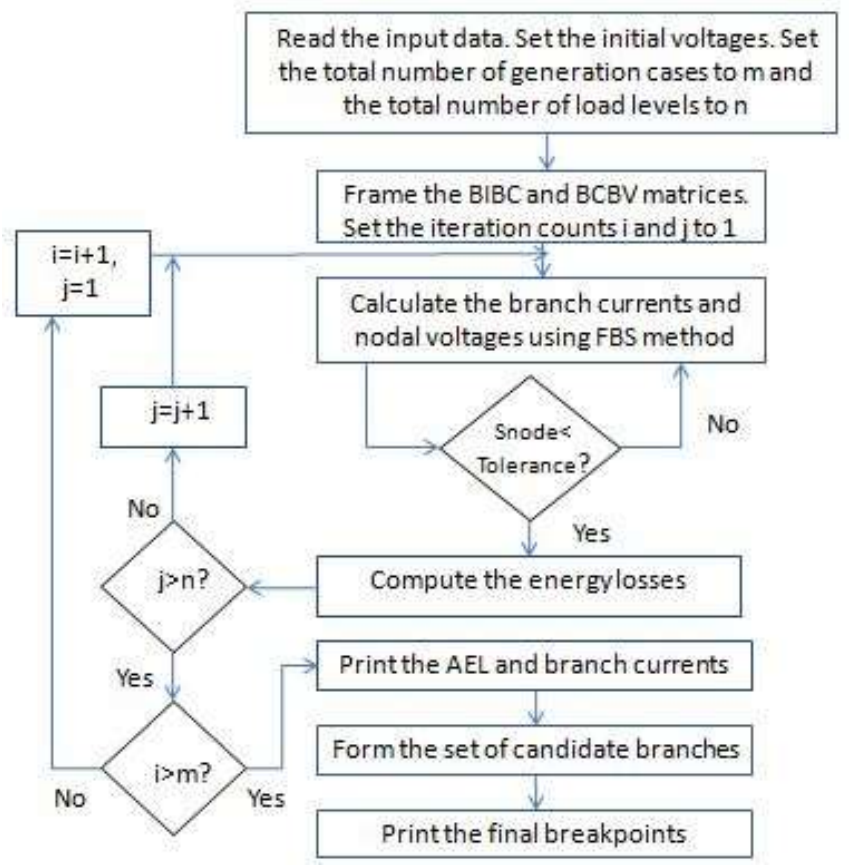

Figure 2. Flowchart for determination of the boundaries of microgrids

Step 3: On reading the real part of $I_{b r}$ for all the cases of the chosen year, increment the counter $\mathrm{x}$ by 1 , when the up-to-down progression of current is found. Likewise, increase the counter y by 1 for every up-to-down flow of $Q_{D G}$. At last, get the summation of all up-to-down flows of $P_{D G}$ and $Q_{D G}$ in the matrices $A$ and $B$, respectively. The maximum values in these matrices will be equal to the total number of cases handled and the minimum value possible is zero. The maximum value for a particular branch implies that the branch always carries up-to-down power. The minimum value for the branch connotes that the branch consistently supplies the grid from downstream.

Step 4: When $A$ and $B$ show a trend reversal of $I_{b r}$, form the set of candidate branches referring to Section 2.

Step 5: The final breakpoints are at last decided from the set of candidate branches using the following constraints:

a) The number of nodes in each microgrid should be at least four.

b) Each microgrid ought to have a sufficient generation to keep up the reliability at fault conditions.

c) In the event that two nearby nodes encounter almost similar trend reversal pattern, the counts $\mathrm{x}$ and $\mathrm{y}$ in $A$ and $B$ are analyzed to determine the stronger breakpoint.

Sometimes, there are possibilities that the algorithm arrives at two different sets of breakpoints, one based on $A$ and the second based on $B$. In such a case, the final decision is made giving priority to either $P_{D G}$ or $Q_{D G}$. Step 6: To find the reliability of the system, refer to Section 3.3. 


\subsection{Optimization problem}

One of the objectives of this paper is to find the optimal percentage of penetration, $P P$ of the DGs corresponding to minimal annual energy loss $(A E L)$, and a maximum number of microgrids. The objective function $F_{L S}$ can be defined as in (3):

$$
\text { Minimize } F_{L S}=\left(Y_{1} \times A E L\right)+\left[Y_{2}(1-N S)\right]
$$

where $N S$ is the total number of splits performed. The maximum number of splits possible is determined by dividing the total number of nodes in the system by MNPM. $Y_{1}$ and $Y_{2}$ are the weights allotted to the two different objectives. At the point when loss minimization is a higher priority than the total number of splits, set $Y_{1}>Y_{2}$ or vice-versa. Here, $Y_{l}=Y_{2}=0.5$ is chosen to give equal importance to the two targets.

The objective in (3) is subjected to the below constraints:

a) The variation of $P P$ of the DGs is based on the uncertain percentages of the load. The load may vary from $L_{\min }$ to $L_{\max }$ in the considered time period. For this time span, using the $P O C$ of each level of load, compute the limits $P P_{\min }$ and $P P_{\max }$, by ignoring the minimal probability cases of the load.

$$
\left.P P_{\min } \leq P P \leq P P_{\max }\right)
$$

b) The voltages of all the buses except slack bus should be within permissible limits, i.e.,

$$
V_{\min } \leq V_{\text {bus }} \leq V_{\max }
$$

Here, $V_{\min }$ is 0.9 p.u. and $V_{\max }$ is 1.05 p.u. whereas, for the slack bus, the voltage is assumed to be $1\llcorner 0$.

Now that the objective and constraints are defined clearly, the exhaustive search method is used in this paper to get the optimal $P P$ as in [20].

\section{RESULTS AND DISCUSSION}

The well-known PG\&E 69-bus radial distribution system that has DGs installed as shown in Table 2 is now chosen to act as the test system. The total $P_{D G}$ requirement of the system is $3802.19 \mathrm{~kW}$ and the total $Q_{D G}$ demand is $2694.6 \mathrm{kVAR}$. The $P P$ of the DGs is kept varying to find the most optimal state of functioning of the system. As the $P P$ increases from a minimum to $100 \%$, the maximum capacities of all the DGs are scaled up, proportionally. All the DGs are capable of supplying both $P_{D G}$ and $Q_{D G}$ to the system. The results obtained using MRCFM are compared with the outcomes acquired using the "Reverse Current Flow Method (RCFM)" in [3].

Table 2. Allocation of DGs for $65 \%$ penetration

\begin{tabular}{ccc}
\hline DG Type & Bus number in which DGs are installed & Maximum $\mathrm{P}_{\mathrm{DG}}$ in $\mathrm{kW}$ \\
\hline Wind turbine & $16,25,46,49,52,64$ & $116,58,174,174,232,58$ \\
Biomass unit & $9,48,51,54$ & $116,290,406,464$ \\
Solar PV & $20,23,32,37,41,56$ & $58,58,116,58,58,58$ \\
\hline
\end{tabular}

\subsection{Optimal state of operation of DGs}

All the possible blends of the load-generation mentioned in Section 3.2 of this paper are considered along with their probabilities of events. The parameters required for the calculation of $Q_{D G}$ are given in [4]. For an SG-based biomass unit, the direct axis and the quadrature axis components of the synchronous reactance is 1.54 p.u. and 0.9 p.u., respectively. The maximum load angle equals $85^{\circ}$ [21]. By differing the infiltration of the DGs from a minimum to $100 \%$, in discrete steps, the values of losses are found using the FBS algorithm for all the 5040 cases and summed up to get the $A E L$ values. The loss calculation procedure, objective statement, and constraints in Section 3 are employed. Figure 3 is a plot between the $P P$ of DGs and $A E L$ values. It is discovered that when the DGs operate to supply a maximum of $65 \%$ of the load, the minimum $A E L$ point occurs. This is the optimal state of operation.

Ranging from lower end to higher end, three discrete $P P$ is now fixed to be equivalent to $28 \%, 65 \%$, and $100 \%$. AEL, voltage magnitude range in p.u., and boundary decision that is made for splitting the chosen distribution system into microgrids are selected to be the comparison parameters, as the $P P$ differs. Table 3 shows the outcomes obtained for the three different percentages. 


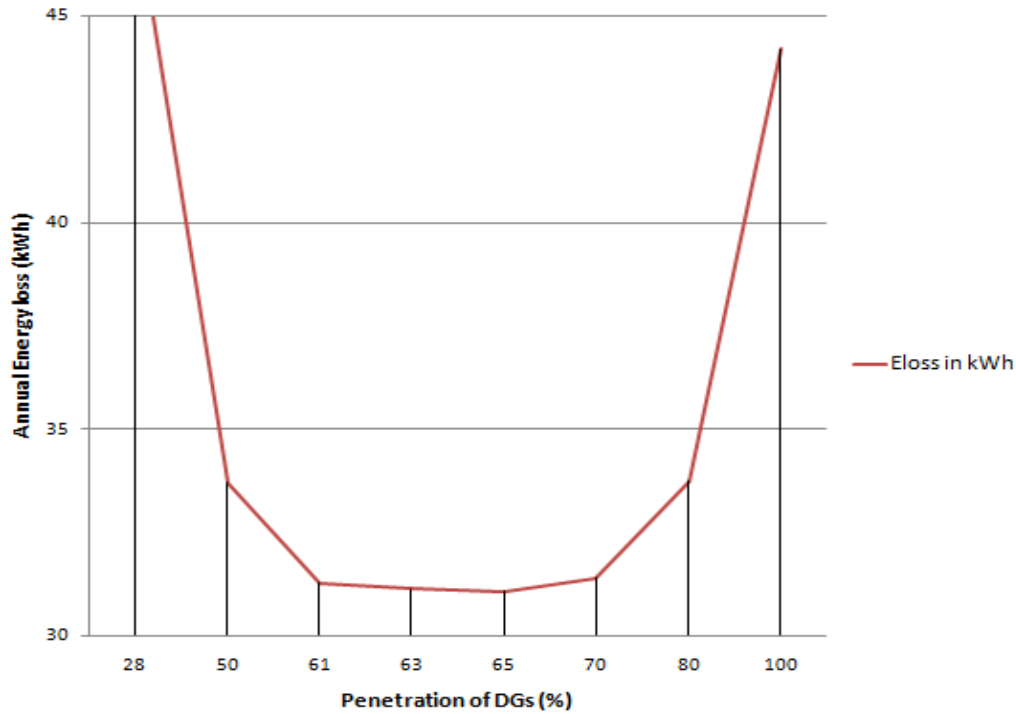

Figure 3. Best percentage of penetration of distributed generation

Table 3. Comparison of results for varying percentages of supply of the DGs

\begin{tabular}{ccccc}
\hline $\begin{array}{c}\text { PP of the } \\
\text { DGs }\end{array}$ & $\begin{array}{c}\text { AEL in } \\
\text { kWh }\end{array}$ & $\begin{array}{c}\text { Voltage magnitude } \\
\text { range in p.u. }\end{array}$ & $\begin{array}{c}\text { Boundary branch } \\
\text { numbers }\end{array}$ & $\begin{array}{c}\text { Number of microgrids in the system after } \\
\text { performing maximum number of splits }\end{array}$ \\
\hline 28 & 47.9 & $0.91-1.01$ & 49 & 2 \\
65 & 31.1 & $0.9-1.05$ & $20,27,49$ & 4 \\
100 & 44.2 & $0.9-1.1$ & 17,49 & 3 \\
\hline
\end{tabular}

It can be seen that AEL is higher for a much lesser or higher PP. To be precise, AEL increases when the distribution system is too dependent on the substation with a much lesser supply from the DGs or when supply from the DGs exceeds the optimal point, leading to the transfer of the excess kVA to the grid upstream. On checking the voltage profile, the same table shows that the limits of voltages are crossed when the infiltration rate rises to $100 \%$. Lastly, on comparing the boundary decisions made for the different PP, it is clear that the maximum number of splits can be performed just when the level of infiltration is at $65 \%$. This is the optimal point of operation, and it gives maximum self-ampleness for the radial distribution system. The pictorial representation of the optimal planning made at $65 \%$ penetration is shown in Figure 4.

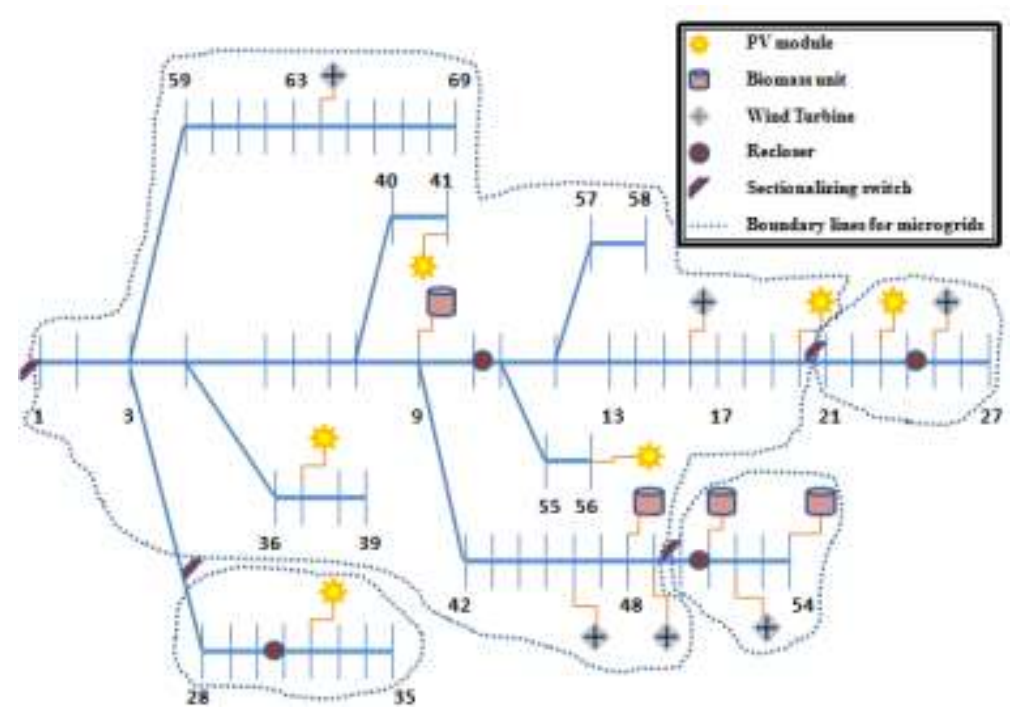

Figure 4. Boundaries of microgrids for $65 \%$ penetration of the DGs-MRCFM 
The boundaries are decided as per the sequence given in Section 3.4. When the rate of infiltration of the DGs is very less, an increased number of splits cannot be performed on the system as it is excessively reliant on the upstream grid. During isolated operation of the microgrids, enough supply will not be available and this will prompt a reduction in the reliability of the system. Similarly, when the supply from the DGs is beyond the optimal point of operation, it is necessitated that the extra kVA is sent to the substation. During faults, the reliability of the independent microgrids will be very high, yet chopping down the supply from the DGs needs to be performed to keep up the voltages inside cutoff points. This is definitely not the preferred solution as the DGs give sustainable power, that is capital-intensive. Hence it is concluded that at $65 \%$, the objective stated in (3) is achieved.

When the values of $Q_{D G}$ are ignored for the splitting problem as found in the literature, $B$ shows that $Q_{D G}$ is constantly obtained from the substation and thus islanding any segment of the grid will bring about loss of reliability. Consequently, a more practical method of calculation of $Q_{D G}$ is employed in this paper.

\subsection{Comparison between MRCFM and RCFM}

RCFM, an approximate method found in [3] has solved the problem for $28 \%$ infiltration of the DGs. The outcomes acquired using MRCFM for the same percentage is now chosen, to compare the methods. RCFM uses the formula $Q_{D G}=\left(P_{D G} \mathrm{x} \tan \delta\right)$, where $\delta$ is the angle of capacitive pf, to get the values of $Q_{D G}$. Whereas, the proposed MRCFM utilizes the P-Q curves and considers both capacitive and inductive pf to get the values of $Q_{D G}$, and proves to be a practical and more accurate method. For $28 \%$ penetration of the DGs, the estimation of $A E L$ equals $44.55 \mathrm{kWh}$, on using the approximate RCFM. For the same case, $A E L$ equals $47.9 \mathrm{kWh}$, on using the more viable and exact MRCFM.

Figure 5 shows the voltage profiles acquired using RCFM and MRCFM for the cases of minimum and maximum generations. The curve for the minimum generation obtained using MRCFM shows a larger drop in voltages at certain nodes as it considers the consumption of $Q_{D G}$ at lagging pf operation. This is again a more practical and exact portrayal of the system when contrasted with the approximate RCFM. The bus voltages are under permissible limits, for all the cases considered under MRCFM.

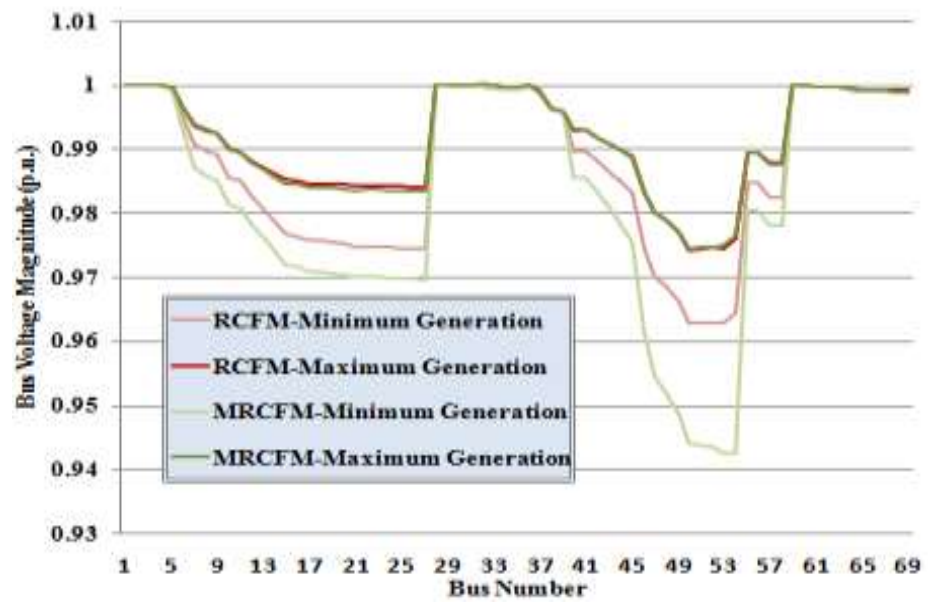

Figure 5. Voltage profile comparison between RCFM and MRCFM

Coming to the splitting sequence devised under RCFM, it is discovered that only the real part of $I_{b r}$ were considered for the splitting operation. Figure 6 exhibits the boundary decision made using RCFM for $28 \%$ penetration of the DGs, and the number of microgrids formed is 5 . When the PP is very high or too low, splitting the distribution system into numerous parts as in Figure 6 affects the reliability of the system, drastically. On the other hand, MRCFM provides better $Q_{D G}$ backing to the grid by considering the imaginary part of $I_{b r}$. MRCFM splitting ends up in a lesser number of microgrids in the system and improves the reliability, unlike RCFM. Figure 7 shows the parting made utilizing MRCFM for the same percentage, and the number of microgrids formed is 2 . The count of protective devices installed in Figure 7 is lesser ( 2 sectionalizers and 3 reclosers) compared to RCFM (5 sectionalizers and 5 reclosers) and this results in considerable economic savings. Subsequently, the calculation of $A E L$, comparison of the voltage profiles obtained, and the boundary decision strategy of MRCFM speak about the superiority of the method over RCFM. 


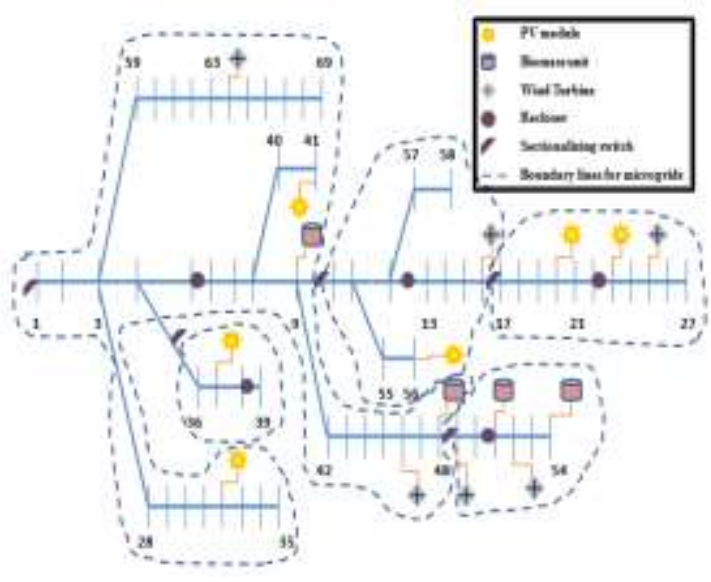

Figure 6. Boundaries of microgrids-RCFM

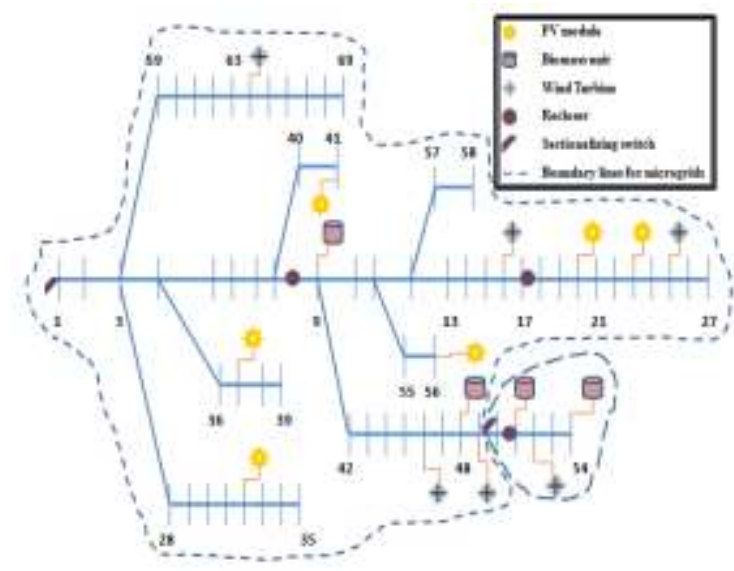

Figure 7. Boundaries of microgrids-MRCFM

\subsection{Estimation of reliability}

Utilizing the input data specified in Table 4, reliability estimation is made for the systems shown in Figure 6 and Figure 7. The feeder data is extrapolated for every line segment referring to [22-24]. Referring to $[22,24,25]$, the generator data is obtained for reliability calculation. It is assumed that the load is at a $65 \%$ level and the penetration of the DGs stays at $28 \%$. Additionally, it is assumed that the DGs give maximum $P_{D G}$ and the corresponding $Q_{D G}$ at a leading pf. The DFIG-based wind turbines are assumed to operate for a slip value of -0.25 . For the instances of sustained faults, it is assumed that reclosers are placed such that a third of the total number of customers connected in a microgrid gets affected. The data for substation, transformer, substation breaker, and sectionalizing switches are taken from $[22,26]$. It is assumed that the reclosers behave in a manner that is similar to that of the breakers. Table 5 compares the indices of RCFM and MRCFM. Here, the distribution of load is not so uniform, and thus, there arises a need to compute both the customer-based and the load-based parameters. It is seen that the values obtained for the system in Figure 7 (MRCFM) are lesser compared to those obtained for the system in Figure 6 (RCFM). This implies that MRCFM brings about a highly reliable solution. Moreover, RTPY with and without DG is found to be lesser for MRCFM compared to RCFM, and this results in considerable savings when it comes to manpower employment.

Table 4. Load details for reliability calculation

\begin{tabular}{ccccccccccccccccccc}
\hline LPN & NOC & T L & LPN & NOC & T L & LPN & NOC & TL & LPN & NOC & TL & LPN & NOC & T L & LPN & NOC & TL \\
\hline 1 & 0 & 0 & 13 & 3 & 10 & 25 & 0 & 0 & 37 & 26 & 97 & 49 & 0 & 0 & 61 & 0 & 0 \\
2 & 0 & 0 & 14 & 3 & 10 & 26 & 5 & 17 & 38 & 125 & 473 & 50 & 1 & 152 & 62 & 8 & 29 \\
3 & 0 & 0 & 15 & 0 & 0 & 27 & 5 & 17 & 39 & 120 & 473 & 51 & 11 & 39 & 63 & 7 & 29 \\
4 & 0 & 0 & 16 & 17 & 55 & 28 & 9 & 32 & 40 & 13 & 49 & 52 & 0 & 0 & 64 & 1 & 2 \\
5 & 0 & 0 & 17 & 10 & 69 & 29 & 10 & 32 & 41 & 1 & 5 & 53 & 95 & 279 & 65 & 0 & 0 \\
6 & 1 & 3 & 18 & 13 & 69 & 30 & 0 & 0 & 42 & 1 & 6 & 54 & 12 & 72 & 66 & 2 & 7 \\
7 & 13 & 50 & 19 & 0 & 0 & 31 & 0 & 0 & 43 & 10 & 33 & 55 & 7 & 22 & 67 & 0 & 0 \\
8 & 23 & 92 & 20 & 1 & 1 & 32 & 0 & 0 & 44 & 8 & 30 & 56 & 7 & 22 & 68 & 13 & 47 \\
9 & 11 & 37 & 21 & 35 & 140 & 33 & 4 & 17 & 45 & 0 & 0 & 57 & 10 & 34 & 69 & 12 & 47 \\
10 & 9 & 34 & 22 & 2 & 6 & 34 & 9 & 24 & 46 & 0 & 0 & 58 & 10 & 34 & & \\
11 & 45 & 178 & 23 & 0 & 0 & 35 & 2 & 7 & 47 & 0 & 0 & 59 & 9 & 32 & \\
12 & 42 & 178 & 24 & 11 & 34 & 36 & 0 & 0 & 48 & 28 & 123 & 60 & 8 & 32 & \\
\hline
\end{tabular}

Table 5. Comparison of reliability indices

\begin{tabular}{cccccccc}
\hline Reliability Indices & SAIDI & SAIFI & ASIDI & ASIFI & LOLP & RTPY with DG & RTPY without DG \\
\hline RCFM & 18.8 & 6 & 18.2 & 5.9 & 0.0021 & 5480 & 1076 \\
MRCFM & 15.8 & 3.3 & 11.6 & 3.4 & 0.0013 & 5120 & 716 \\
\hline
\end{tabular}

\section{CONCLUSION}

The objective of this paper was to present a new strategy to split a large radial distribution system into several self-adequate and highly reliable microgrids based on the directions of the flow of the branch currents of the system, ensuring maximum utilization of the distributed generation. On using the novel 
strategy, this study has identified that the optimal point of operation occurs at $65 \%$ penetration of distributed generation, wherein the energy losses were minimum and the number of microgrids formed was maximum. This paper has argued that the proposed "Modified Reverse Current Flow Method" is superior when compared to the existing "Reverse Current Flow Method". The suggested novel technique gave a more precise energy loss estimation and voltage profile. Furthermore, the formation of the microgrids using the new method has improved the reliability of the system, apart from exhibiting monetary benefits related to manpower deployment and placement of the protective devices.

\section{REFERENCES}

[1] Arefifar, S.A., Mohamed, Y.A.-R.I., and El-Fouly, T.H.M., "Supply Adequacy-Based Optimal Construction of Microgrids in Smart Distribution Systems," IEEE Trans. Smart Grid, vol. 3, no. 3, pp. 1491-1502, 2012.

[2] Nassar, M.E., and Salama, M.M.A., "Adaptive Self-Adequate Microgrids Using Dynamic Boundaries," IEEE Trans. Smart Grid, vol. 7, no. 1, pp. 105-113, 2016.

[3] Kavitha, S., Jayashree, R., Mohamed Rafeequdin, I., and Karthikeyan, D., "Defining the Boundaries of Microgrids in A Large Distribution System Ensuring Supply Security," Proc. 2017 Int. Conf. on Power Syst. (ICPS), Pune, India, pp. 277-282, 2017.

[4] Zou, K., Agalgaonkar, A.P., Muttaqi, K.M., et al., "Distribution System Planning With Incorporating DG Reactive Capability and System Uncertainties," IEEE Trans. Sust. Energy, vol. 3, no. 1, pp. 112-123, 2012.

[5] Konopinski, R.J., Vijayan, P., and Ajjarapu, V., "Extended Reactive Capability of DFIG Wind Parks for Enhanced System Performance," IEEE Trans. Power Syst., vol. 24, no. 3, pp. 1346-1355, 2009.

[6] Wang, Z., Zhang, L., and Li, G., "The Active Power and Reactive Power Dispatch Plan of DFIG Based Wind Farm Considering Wind Power Curtailment," Int. J.Comput. Intell. Syst., vol. 8, no. 3, pp. 553-560, 2015.

[7] Z.B.A. Hamid, et al., "Optimal sizing of distributed generation using firefly algorithm and loss sensitivity for voltage stability improvement," Indo. J. Electr. Engi. Comp. Sc., vol. 17, no. 2, pp. 720-727, 2020.

[8] IEEE Standard: "IEEE Guide for Electric Power Distribution Reliability Indices," 2012.

[9] H. Suyono, et al., "Enhancement of the power system distribution reliability using ant colony optimization and simulated annealing methods," Indo. J. Electr. Engi. Comp. Sc., vol. 17, no. 2, pp. 877-885, 2020.

[10] Al-Muihani, M., and Heydt, G.T., "Evaluating Future Power Distribution System Reliability Including Distributed Generation," IEEE Trans. Power Deliv., vol. 28, no. 4, pp. 2264-2272, 2013.

[11] Jung-Uk, L., "Probabilistic Q-Margin Calculations Considering Dependency of Uncertain Load and Wind Generation," Int. J. Appl. Power Engi., vol. 2, no. 3, pp. 141-148, 2013.

[12] Moreno, R., Obando, J., and Gonzalez, G., "An integrated OPF dispatching model with wind power and demand response for day-ahead markets," Int. J. Electr. Comp. Engi., vol. 9, no. 4, pp. 2794-2802, 2019.

[13] Atwa, Y.M., and El-Saadany, E.F., "Probabilistic approach for optimal allocation of wind-based distributed generation in distribution systems," IET Renew. Power Gener., vol. 5, no. 1, pp. 79-88, 2011.

[14] Salkuti, S.R., "Multi-objective based economic environmental dispatch with stochastic solar-wind-thermal power system," Int. J. Electr. Comp. Engi., vol. 10, no. 5, pp. 4543-4551, 2020.

[15] Khormandichali, S.M.M., and Kamarposhti, M.A., "Optimal placement of wind generation units in order to increase revenues and reduce the imposed costs in the distribution system considering uncertainty," Int. J. Electr. Comp. Engi., vol. 9, no. 6, pp. 4524-4539, 2019.

[16] Soroudi, A., and Afrasiab, M., "Binary PSO-based dynamic multi-objective model for distributed generation planning under uncertainty," IET Renew. Power Gener., vol. 6, no. 2, pp. 67-78, 2012.

[17] Kadom, H.F., Hussain, A.N., and Al-Jubori, W.K.S., "Dual technique of reconfiguration and capacitor placement for distribution system," Int. J. Electr. Comp. Engi., vol. 10, no. 1, pp. 80-90, 2020.

[18] Ali, M.H., Mehanna, M., and Othman, E., "Optimal planning of RDGs in electrical distribution networks using hybrid SAPSO algorithm," Int. J. Electr. Comp. Engi., vol. 10, no. 6, pp. 6153-6163, 2020.

[19] P. Ravi Babu, and M. Srivani, "AACO technique for solving multiobjectives in electrical distribution system," Indo. J. Electr. Engi. Comp. Sc., vol. 14, no. 3, pp. 1076-1086, 2019.

[20] López, P. R., Galán, S. G., Reyes, N. R., and Jurado, F., "A Method for Particle Swarm Optimization and its Application in Location of Biomass Power Plants," Int. J. Green Energy, vol. 5, no. 3, pp. 199-211, 2008.

[21] Lof, P.A., Andersson, G., and Hill, D.J., "Voltage dependent reactive power limits for voltage stability studies," IEEE Trans. Power Syst., vol. 10, no. 1, pp. 220-228, 1995.

[22] Zou, K., Agalgaonkar, A.P., Muttaqi, K.M., et al., "An Analytical Approach for Reliability Evaluation of Distribution Systems Containing Dispatchable and Nondispatchable Renewable DG Units," IEEE Trans. Smart Grid, vol. 5, no. 6, pp. 2657-2665, 2014.

[23] Brown, R.E., Gupta, S. Christie, R.D., et al., "Distribution System Reliability Assessment: Momentary Interruptions and Storms," IEEE Trans. Power Deliv., vol. 12, no. 4, pp. 1569-1575, 1997.

[24] Billinton, R., and Allan, R.N., "Reliability Assessment of Large Electric Power Systems," Kluwer Academic Publishers, Boston, 1st edn., 1988.

[25] Singh, C., and Lago-Gonzalez, A., "Reliability Modeling of Generation Systems including Unconventional Energy Sources," IEEE Trans. Power Appar. and Syst., vol. 104, no. 5, pp. 1049-1056, 1985.

[26] Allan, R.N., Billinton, R., Sjarief, I., et al., "Reliability Test System for Educational Purposes-Basic Distribution System Data and Results," IEEE Trans. Power Syst., vol. 6, no. 2, pp. 813-820, 1991. 